\title{
Contribution of microRNAs to CLL Biology and Their Potential as New Biomarkers
}

\author{
Maria Rosa Garcia-Silva ${ }^{1}$, Maria Catalina Güida ${ }^{1}$ and Alfonso Cayotat,2 \\ ${ }^{1}$ Institut Pasteur de Montevideo, \\ ${ }^{2}$ Faculty of Medicine, Montevideo, \\ Uruguay
}

\section{Introduction}

After the complete description of the human genome (approximately $3 \times 10^{9}$ bases) the best estimates of protein-coding genes account for about 30,000 to 40,000 genes representing approximately $1 \%$ of the genome. A significantly remainder fraction of the genome is transcribed into RNAs that do not code for proteins which are classified as non-coding RNAs (ncRNAs) (Wright et al., 2001). These ncRNAs were unnoticed in the genome until recent improvements in high-throughput technology for gene expression assays led to the discovery that most human transcriptional units are ncRNAs. These ncRNAs have been segregated into two main classes; long and small non-coding RNAs. Over the last almost two decades, the family of small ncRNAs (i.e. microRNAs, siRNAs and piRNAs) has grown in number and relevance and emerged as new key regulators of gene expression. These small ncRNAs, which are 19-32 nucleotides (nt) in length, act as sequence-specific triggers for mRNA degradation, translation repression, heterochromatin formation and genome stability affecting biological functions either by posttranscriptional silencing or stimulating transcript degradation. The most well known small ncRNAs are the microRNAs (miRNAs). To date, more than 1500 human miRNAs genes have been annotated. They are organized as mono- or policystronic transcriptional units in the genome located either in intergenic regions or within introns and exons of non-coding as well as coding transcription units (miRBase, release 18: November 2011).

\subsection{Biogenesis and function of microRNAs}

The defining features of these small silencing RNAs are, in addition to their short size, their association with members of the Argonaute family of proteins, which guide them to their regulatory targets. Biogenesis and effector functions of miRNAs require several complex steps (Figure 1). Most miRNAs are transcribed by the RNA Polymerase II as long primary transcripts (pri-miRNAs) with a 5'm7G cap and a 3' poly-A tail. In humans, they are subsequently cleaved in the nucleus by an RNase III endonuclease, Drosha, assisted by a dsRNA-binding protein (DGCR8) to produce a stem-loop precursor of $\approx 70 \mathrm{nt}$ in length (premiRNA). Pre-miRNAs are translocated to the cytoplasm by the Exportin-5/Ran complex and further processed by the cytoplasmic RNase III, Dicer, yielding $\approx 22$-nt duplexes. Several dsRNA-binding accessory proteins assist human Dicer enabling both RNA 
unwinding and loading onto effector complexes containing members of the Argonaute family of proteins as key components (Chu \& Rana, 2007). This complex (miRNA-induced silencing complex or miRISC) is loaded with the mature miRNA while the complementary "passenger" strand is degraded.

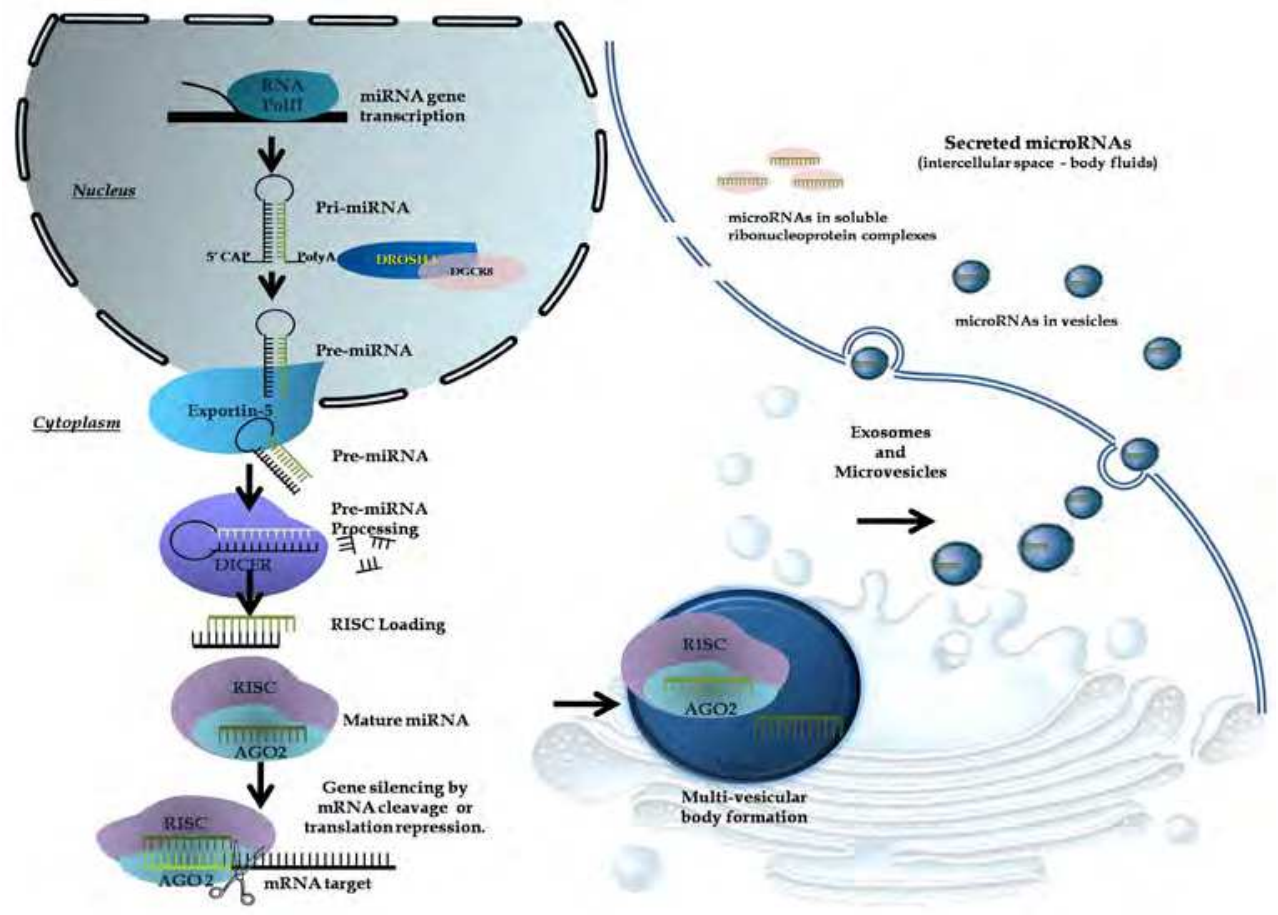

Fig. 1. miRNA biogenesis and effector pathways.

MicroRNAs are initially transcribed by RNA polymerase II as a primary (pri)-miRNA in the nucleus. Binding of the pri-miRNA to DGCR8 and Drosha results in the processing of the pri-miRNA to a $70 \mathrm{nt}$ precursor miRNA (pre-miRNA). This complex is then transported to the cytoplasm by a complex consisting mainly of exportin-5. In the cytoplasm, a pre-miRNA processing complex containing a RNase III endonuclease (Dicer) at its core is formed cleaving the pre-miRNA to a double stranded of $\approx 22 \mathrm{nt}$ miRNA molecule. The miRNA is then separated into two single-stranded molecules; the antisense strand is incorporated in the RNA-induced silencing complex (RISC) through its interaction with Argonaute (AGO) proteins while the other strand is degraded. The mature miRNA is then transported to either the $3^{\prime}$ or $5^{\prime}$ untranslated region of the target mRNA (UTR) for mRNA degradation of inhibition of translation. As a parallel pathway, the loaded RISC is linked to the Multivesicular bodies (MVB) and could be secreted to the extracellular medium an endocytosed by other cells. A significant fraction of miRNAs are secreted as ribonucleoprotein complexes. 
This loaded complex is the effector responsible for the gene silencing of the mRNA target. Post-transcriptional gene silencing mediated by miRNAs involves their binding by partial or full complementarity to specific regions or binding sites on untranslated regions (UTRs) of target mRNAs. The commonly accepted mechanism of miRNA targeting in animals involves an interaction between the 5'-end of the miRNA called the "seed region" (about 7 nts) and the $3^{\prime}$ untranslated region (3'-UTR) of the mRNA. This binding induces either translation inhibition or cleavage of target mRNAs.

\subsection{Expression patterns of microRNAs}

Numerous miRNAs exhibit characteristic expression patterns that could serve as a fingerprint of a particular tissue, cell type, biological state, etc. Some miRNAs are differentially expressed in developmental stages, like the first family members, lin- 4 and let7 in C. elegans. For this reason, they were called at the beginnings stRNA (small temporal RNA) because they are expressed in specific temporal phases of development and regulate the correct developmental timing. In mammalian cells, a miRNA expression pattern can usually be related to its possible role. Analogous to mRNA expression, miRNA expression is determined by intrinsic cellular factors as well as diverse environmental variables. The study and characterization of miRNAs was delayed several years due to their small size. However, the recent development of small RNA-adapted cloning, hybridization technologies and sequencing protocols allowed the use of high-throughput sequencing, microarrays and real time PCRs to characterize small RNAs in various genomics studies. Taken as a whole these technical improvements are expected to greatly extend the collection of miRNAs in a variety of biological systems.

\subsection{MicroRNAs as key regulators of gene expression}

It has become clear that miRNAs confer a novel layer of post-transcriptional regulation through fine-tuning gene expression, which is widely used in plants and animals. They are estimated to comprise $1 \%-5 \%$ of animal genes making them one of the most abundant classes of gene expression regulators. An increasing body of experimental data and bioinformatics prediction of miRNA targets revealed that miRNAs are expected to regulate more than $30 \%$ of protein coding genes (Croce, 2009). Most genes involved in basic processes common to all cells are under selective pressure to avoid miRNA-mediated regulation ("antitargets"). In contrast, many genes involved in developmental processes, cell proliferation, apoptosis, metabolism, cell differentiation, and morphogenesis ("targets") are enriched in miRNA binding sites by changes in 3' UTR (and more recent discovered also in 5' UTR) length and density during evolution (Farazi et al., 2011). These data induced many authors to speculate about a putative role of miRNAs in cancer and other human pathologies.

\subsection{MicroRNAs as new actors in cancer biology}

Nowadays, it is widely accepted that miRNAs could promote or suppress malignant processes in a similar manner to classical oncogenes and tumor suppressors. In the first case, miRNAs targeting mRNAs encoding for proteins that promote tumor initiation and progression are classified as tumor suppressor miRNAs. Thus, the loss of function of a tumor suppressor miRNA by genomic deletion, mutation, epigenetic silencing, and/or 
miRNA processing alterations ultimately leads to an inappropriate increase in levels of the respective mRNA target, which in turns initiate or contribute to the malignant transformation. On the other hand miRNAs are classified as oncogenes when their target mRNAs code for tumor suppressor proteins. Overexpression or amplification of these miRNAs is followed by down-modulation of the target tumor suppressor protein, which ultimately initiates or contributes to the malignant transformation (Rovira et al., 2010).

The levels of miRNAs usually are precisely controlled in the cells to guarantee a correct cell life cycle, function and differentiation. It was commonly observed that aberrant expression of miRNAs was associated to malignant transformation. The first description of a miRNA associated to cancer was reported in CLL by Calin et al (Calin et al., 2002). It is known that the most frequent chromosomal alteration, identified in more than $50 \%$ of patients suffering from B-cell Chronic Lymphocytic Leukemia, is a deletion of chromosome 13q14 that associated with longer survival. Deletions at $11 q$ and $17 p$ are also typical, although much less frequent, and correlate with up-regulation of the zeta-chain-associated protein kinase $70 \mathrm{kDa}$ (ZAP70) and a shorter overall survival. These findings led to extensive efforts to define a putative tumor suppressor gene or genes at the $13 \mathrm{q} 14$ locus. No plausible proteinencoding candidate was identified into this region. A perceptive change in the strategy for possible regulatory elements along this region unearthed the existence of two novel miRNAs; miR-15a and miR-16-1 (Calin et al., 2002). This was the first indication that miRNAs could function as tumor suppressors showing frequent deletions or downmodulation of miR-15a/16-1 in patients suffering from CLL, and the first link between miRNAs and cancer. A second report from this lab showed that about $50 \%$ of annotated human miRNAs are located in "fragile sites" of the genome frequently associated with cancer (Calin et al., 2004b). Further studies found that these related miRNAs have as target the mRNA encoding the antiapoptotic protein B-cell CLL/lymphoma 2 (BCL2), the upregulation of which is critical for CLL cell survival (Cimmino et al., 2005). Importantly, the direct interaction of $m i R-15 / m i R-16$ with BCL2 transcripts delayed protein translation, induced apoptosis, and reinforced the role of miRNAs as part of a new class of tumor suppressor genes.

\section{MicroRNAs reported in the initiation and progresion of CLL}

The implication of miR-15a/16-1 in CLL patients described by Calin et al. and other fascinating results, led to Corney et al. (Corney et al., 2007) to explore the potential influence of the well known tumor protein p53 (TP53) on miRNA regulation. This work revealed that TP53 directly activates the expression of the $m i R-34 b / m i R-34 c$ cluster situated at $11 \mathrm{q}$, which is proximal to the region deleted in patients with CLL who have poor outcomes. Along with the noticeable location of the TP53 gene at 17p, these findings were suggestive of a higher-order genetic connection in CLL pathogenesis.

These findings led to a rapidly expanding series of investigations linking miRNAs to CLL. As a result, miRNAs are currently under evaluation as novel putative diagnostic and prognostic biomarkers as well as potential therapeutic targets in CLL. Consequently, differential miRNA signatures distinguishing between tumor and normal tissues were reported in leukemia and solid tumors. Several recent reports suggested a miRNA signature associated with diagnosis, prognosis and progression of CLL. 
As mentioned above, Calin et al. conducted a miRNA expression profiling on a wellannotated cohort of 94 CLL patients and identified a panel of 13 miRNAs that correlated with ZAP-70 expression and $\mathrm{I}_{\mathrm{g}} \mathrm{V}_{\mathrm{H}}$ mutational status (Calin et al., 2005). In addition, a subgroup of nine miRNAs $(181 b, 155,146,24-2,23 b, 23 a, 222,221$, and 29c) differentiated patients with a short interval to therapy from patients with a longer interval to therapy. These authors also identified germ line or somatic mutations in miRNA genes in 15\% of CLL patients studied. miRNA expression in B cells from a cohort of 50 CLL patients identified 7 upregulated miRNAs and 19 downregulated miRNAs (Calin et al., 2005). Similar to this previous study, the authors identified the upregulation of $m i R-155$ and downregulation of $m i R-181 a / b$. Although several studies have demonstrated a correlation between chromosomal alterations and miRNA deregulation, these studies failed to demonstrate such a connection, so the questions in this topic remain to be answered. Gain of methylation was present in pri-miRNAs for several deregulated miRNAs, including miR-139 and miR-582. Thus, this suggests that epigenetic regulation is likely to have a role in altered miRNA expression in CLL (Nana-Sinkam \& Croce, 2010).

Interestingly, it was recently reported (Fabbri et al., 2011) that the recurring deletion hot spots at 13q, 11q, and 17p actually represent nodes of a complex regulatory network in CLL that integrates the miR-15a/miR-16-1 and miR-34b/miR-34c clusters with the tumor suppressor p53. These studies have lighted a comprehensive hypothesis of CLL pathogenesis that makes a relationship between clinical heterogeneity, complex cytogenetic patterns and prognostic markers. The critical interactions of anti-apoptotic factors such as BCL2, the p53 tumor suppressor, and the ZAP-70 tyrosine kinase, all governed by miRNAs that derive from the long arms of chromosomes 13 and 11, may therefore be involved in managing the variety of indolent or aggressive phenotypes experienced by patients with CLL.

Different studies were performed to identify miRNAs profiles defining leukemic cells involved in CLL. Using different experimental approaches (i.e. qRT-PCR, cloning and microarrays of defined miRNAs) several groups reported at least 25 miRNAs that were differentially expressed in CLL cells versus normal cells. These miRNAs included miR-16-1, miR-26a, miR-206, and miR-223 (Calin et al., 2004a), miR-155, miR-21, miR-150, miR-92 and miR-222 (Fulci et al., 2007) and miR-181, miR-30d, let-7a and three newly reported miRNAs (miR-1201, miR-1202 and miR-1203) characteristics of the CLL cells (Marton et al., 2008).

Several reports identified miRNA signatures that could act as surrogate prognostic biomarkers in CLL, typically by correlating expression levels of these miRNAs with previously established prognostic markers such as $\mathrm{IgV}_{\mathrm{H}}$ mutation status or ZAP-70 expression (Calin et al., 2005). This signature included miR-15a, miR-195, miR-221, miR-23b, miR-155, miR-223, miR29a-2, miR-24-1, miR-29b-2, miR-146, miR-16-1, miR-16-2, and miR29c. Some authors have also developed a quantitative RT-PCR score combining miR-29c, miR-223, ZAP-70 and lipoprotein lipase (Stamatopoulos et al., 2009). Over-expression of miR-21 and low miR-181b expression has been reported as unfavorable prognostic factors independent of other clinical-pathologic factors (Rossi et al., 2010).

The rapid development of miRNA research in the past few years suggests that the roles of many more miRNAs in CLL have yet to be discovered. For instance, for some researchers, the miR-17-92 cluster is a group of miRNAs that have been studied in a wide variety of 
cancers ((Ward et al., 2011)). This miR-17-92 cluster consists of seven miRNAs: miR-17-5p, miR-17-3p, miR-18, miR-19a, miR-20, miR-19b-1 and miR-92-1 transcribed from the MIR17 Host Gene (MIR17HG) at locus 13q31.3, and members of this cluster are thought to coexpress with the proto-oncogenic transcription factor MYC (He et al., 2005). Several profiling studies show that expression of members of the miR-17-92 cluster is altered to some degree in CLL (Calin et al., 2004a; Fulci et al., 2007). Thus, advances in miRNA biology will likely have an increasing influence in the diagnosis, prognosis and treatment of human cancers, including CLL. In addition, it is discussed below the discovery of the oncogenic and tumor-suppressive properties of various miRNAs that come up with the possibility of miRNA therapy for cancer in the near future.

\subsection{The miR-15a/16-1}

The association of miR-15a/16-1 in the pathogenesis of CLL was not only the first implication of miRNAs in this disease, but also in cancer. The authors of this finding were Calin et al. who determined that miR-15a/16-1 were located in 13q14.3, and were either deleted or down-regulated in $68 \%$ of patients with CLL (Calin et al., 2002).

As this chromosomal region is also deleted in other types of cancer (mantle cell lymphoma, multiple myeloma, DLBCL, mature T-cell lymphoma, and solid tumors) conducted several authors to search for the presence of tumor suppressor genes in this region. A region of more than $1.0 \mathrm{Mb}$ was sequenced, but none of the protein genes identified were found implicated in the initiation or progression of CLL (Calin et al., 2005). That fact, give rise to some hypothesis that finally conducted authors to search for miRNAs candidates, unknown genes, or possibly, extremely small genes perhaps not detected by classic cloning methods that might reside in this region and be the real target of genomic alteration (Aqeilan et al., 2010). On this way, a minimal deleted region (MDR) that contains two tightly linked miRNAs, miR-15a and mirR-16-1 was identified (Calin et al., 2002; Lagos-Quintana et al., 2001; Migliazza et al., 2000) (Figure 2). Recent studies demonstrated that other genes located in the same region (deleted in leukemia gene, DLEU 2 and 7), may also function as tumor suppressors (Palamarchuk et al., 2010).

In addition to chromosomal abnormalities, other mechanisms as mutation, loss of heterozygosis, epigenetic deregulation and defects in the miRNA biogenesis machinery, could also contribute to deregulation of miRNA expression (Deng et al., 2008). In this respect, Calin et al. demonstrated that mutations in miR-16-1 could be responsible for the altered expression observed in CLL patients compared with subjects without cancer (Aqeilan et al., 2010).

Using bionformatic tools Cimmino et al. (Cimmino et al., 2005) found that miR-15a and miR-16- sequences share complementary homology with BCL2 (B-cell CLL/lymphoma 2) mRNA sequence. $\mathrm{Bcl} 2$ is an anti-apoptotic protein that is highly expressed in CLL as in other types of human cancer, including leukemias, lymphomas, and carcinomas (Sanchez-Beato et al., 2003)

As the BLC2 gene is overexpressed and that deletions or down-regulation of the miR-15a and miR-16-1 cluster have been reported to occur in the same proportion in CLL samples, it was proposed that miR-15a and miR-16-1 produce their anti-tumorigenic effect by targeting the BCL2 gene (Aqeilan et al., 2010; Cimmino et al., 2005). Its function has also been assayed 
in vitro and in vivo. In immunocompromised nude mice, ectopic expression of miR-15a/16-1 was found to cause dramatic suppression of tumorigenicity of MEG-01 leukemic cells exhibiting a loss of endogenous expression of miR-15a/ 16-1 (Calin et al., 2008) On the other hand, and besides the results obtained by Cimmino et al, other authors found no correlation between BCL2 and mir15a and miR-16-1 in cancer (Fulci et al., 2007; Hanlon et al., 2009; Klein et al., 2010; Linsley et al., 2007). Klein et al. (Klein \& Dalla-Favera, 2010) confirmed the observation that the miR-15a/miR-16-1 locus controls B cell expansion by modulating proliferation, rather than influencing survival via regulation of BCL2 in vivo.

Nowadays, different methodological approaches revealed that CCND1 (encoding cyclin D1) and WNT3A mRNAs which promote several prostate tumorigenic features, could also be directly affected by miR-15a/16-1 (Bonci et al., 2008), like other cancer genes such as MCL1, ETS1 and PDCD6IP that directly or indirectly affect cell cycle and apoptosis.

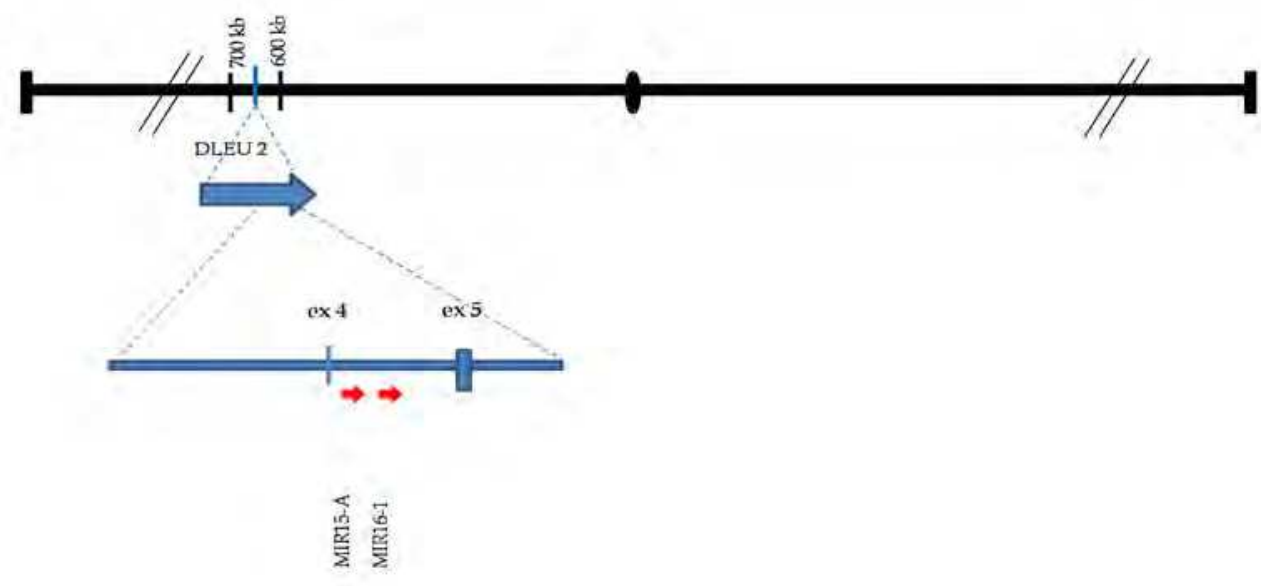

Fig. 2. Localization of miR15/16 cluster on human chromosome 13.

The 13q14 tumor suppressor locus deleted in CLL contain DLEU 2 gene where MIR15a/MIR16/b is located inside, between exon 4 and 5 .

\subsection{The miR-34 family}

The miR-34 family has been implicated in several solid and hematological malignancies. The three members of the miR-34 family are encoded by two different genes: miR-34a is encoded by its own transcript, whereas miR-34b and miR-34c share a common primary transcript (Auer et al., 2007; Cole et al., 2008).

Deregulation of the miR-34a and miR-34b/c expression by chromosomal deletion and/or epigenectic inactivation, presumably occurs during tumorigenesis (Hermeking, 2010).

In the case of miR-34a, chromosomal deletion of the region in which it resides (locus 1p36) and epigenetic inactivation were identified in tumors. Moreover the epigenetic inactivation 
of miR-34a was identified in cell lines derived from some of the most common tumors and in primary melanoma. In addition, CpG methylation of miR-34b/c was also found in colorectal cancer, in oral squamous cell carcinoma and in malignant melanoma.

In the case of CLL, the variability of the mir-34a expression observed in patients is not precisely associated to the just mentioned process. It was demonstrated that members of the miR-34 family are direct p53 targets. MiR-34 genes are up-regulated by the tumor-suppressor protein p53, and their overexpression in turn causes senescence, apoptosis, or cell cycle arrest by regulating proteins such as BCL2, Cyclin D1, Cyclin E2, CDK4, and c-MYC Sirt-1, depending on the cell type (Corney et al., 2007; Ward et al., 2011). Further analysis effectively determined that miR-34a expression partially correlated with p53 status and patients with p53 mutations or deletions of 17p13.1, in general had lower miR-34a expression (Figure 3). However, in some patients decreased miR-34a was seen without p53 aberrations. These patients are homozygous for the single nucleotide polymorphism 309 (SNP309) in the intronic promoter of MDM2, a negative regulator of p53 (Asslaber et al., 2010). The down-regulation of miR-34 b/c is also related to a p53 inactivation and to one of the most characteristic chromosomal deletion in CLL: 11q23.1, where the miR-34 b/c cluster is located.

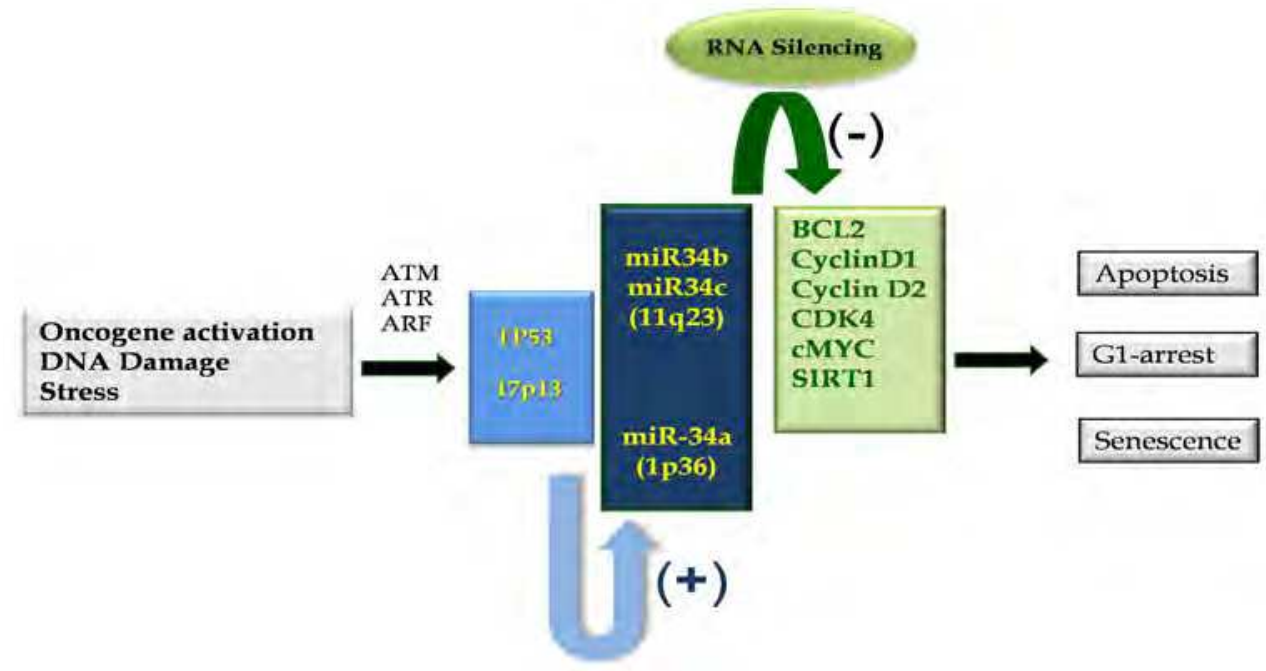

Fig. 3. MicroRNA-34 family regulation and targets involved in CLL. microRNA 34b (miR$34 b) /$ microRNA 34c $(m i R-34 c)$ cluster and miR-34a are regulated by tumor suppressor protein p53. After DNA damage or cellular stress, p53 is activated through ATM, ARF or ATR pathways and transactivates target genes including the miR-34 family members. The mature transcripts of the activated miR-34a/b/c induce either translation inhibition or cleavage of the indicated RNA targets.

\subsection{The miR-29 and miR-181 in CLL}

The members of miR-29 family are arranged in two different loci; the miR-29b-1/miR-29a located at $7 \mathrm{q} 32$ and the miR-29b-2/miR-29c at $1 \mathrm{q} 32$. 
Downregulation of miR-29 members have been reported in various human cancers including aggressive chronic lymphocytic leukemia (Garzon et al., 2009). They were demonstrated to have a tumor suppressor activity by targeting several oncogenes as the Tcell leukemia/ lymphoma 1 (TCL1), the BCL2 family member MCL1, the cyclin-dependent kinase CDK6, and the transcriptional repressor Yy1 (Mott et al., 2007; Pekarsky et al., 2006; Zhao et al. 2010).).

Fabbri et al, showed that members of the miR-29 family target also the de novo DNA methyltransferases (DNMTs) and can reactivate tumor suppressor genes (Fabbri et al., 2007). Thus, loss of miR-29 family members could cause epigenetic changes associated with CLL and other cancer types.

Besides of the antitumoral activity of miR-29 overexpression by inhibition of cell proliferation, it was also observed that miR-29 up-regulation could also initiate acute and chronic leukemias in animal models. Santanam and co-workers (Santanam et al., 2010) developed a transgenic mice overexpressing mir-29 in mouse B cells. They reported that miR-29 is overexpressed in indolent CLL compared with normal B cells. In contrast, miR29 was down-regulated in aggressive CLL.

In addition, it was demonstrated that miR-29 inversely correlated with levels of TCL-1 in CLL patients. TCL-1 is a coactivator of AKT, an oncogene that inhibits apoptotic patways and has a critical role in the regulation of many relevant cell processes including cell proliferation and cell death (Santanam et al., 2010; Vasilatou et al., 2010). Taken together, these data led authors to hypothesize that TCL1 is mostly not expressed in indolent CLL and probably does not play an important role in this disease stage. Indeed, miR-29 overexpression is not sufficient to initiate aggressive CLL. In contrast, up-regulation of TCL1 is a critical event in the pathogenesis of the aggressive form of CLL and because miR-29 is down-regulated in aggressive CLL (compared with the indolent form), it contributes to the development of an aggressive phenotype (Santanam et al., 2010). As mir-29b, mir-181b acts as tumor-suppresor in aggressive CLL by targeting also the TCL-1 oncogene, there is an inverse correlation between TCL1 and miR-181 expression at different stages of B-cell development (Pekarsky et al., 2006).

The high expression of these miRNAs is associated with expression of unmutated $\operatorname{IgV}_{\mathrm{H}}$ and high expression of ZAP-70, indicating an aggressive CLL phenotype (Vasilatou et al., 2010).

\subsection{Other miRNAs potentially implicated in CLL pathogenesis}

As was previously described in this chapter, several miRNAs have been reported to be differently expressed in B cells from CLL compared to normal B-cells.

The miR-155, for example, was reported overexpressed in solid tumors, including lung, colon, and breast cancer as well as in both acute myeloid leukemia and CLL (Garzon et al., 2009). Frenquelli et al. (Frenquelli et al., 2010) recently showed an inverse relationship between miR-221/222 and p27 expression and validated p27 as a functional target for miR221/222 in CLL. Fulci and co-workers (Fulci et al., 2007) found an overexpression of miR150, miR-223, miR-29b, and miR-29c in CLL patients with a mutated $\operatorname{IgV}_{\mathrm{H}}$ phenotype compared to the patients with unmutated $\operatorname{Ig} V_{H}$ phenotypes. 


\section{MicroRNA signatures and prognostic miRNAs in CLL}

In addition to the well known molecular factors (mutational status of $I g V_{H}$, expression levels of ZAP-70 or $\beta 2$-microglobulin and expression of CD38+) and chromosomal abnormalities $(11 q, 13 q, 17 p$ deletions) associated to the clinical course of CLL, the molecular basis for these correlations was largely unknown. However, several studies identified miRNAs that could act as prognostic indicators in CLL, typically by correlating expression levels of these miRNAs with previously mentioned established prognostic indicators (Ward et al., 2011).

One of the first works that described this relationship was published by Calin et al. in 2005 . They described a signature of 13 miRNAs (miR-15a, miR-195, miR-221, miR-23b, miR-155, miR-223, miR29a-2, miR- 24-1, miR-29b-2, miR-146, miR-16-1, miR-16-2, and miR-29c) differentially expressed between unmutated $\mathrm{IgV}_{\mathrm{H}} / \mathrm{ZAP70}+$ and mutated $\mathrm{IgV}_{\mathrm{H}} / \mathrm{ZAP70}$ CLL patients. Further studies, using different molecular techniques, also confirmed the mentioned correlation (Fulci et al., 2007; Marton et al., 2008; Rossi et al., 2010; Stamatopoulos et al., 2009). They reveled also the implication of miR-150 and miR-181 as prognostic factors.

It was recently reported that the deregulation of miR-181b expression can be monitored throughout the course of the disease, which correlate with the overexpression of 4 genes with great significance in CLL biology and other cancers (i.e. MCL1, TCL1, BCL2 and AID (Visone et al., 2009).

An interesting link between the classical prognostic molecular and chromosomal markers of CLL and two groups of defined miRNAs have been recently described by Fabri et al. (Fabbri et al., 2011). They found that miR-15a/miR-16-1 cluster is associated with reduced expression levels of TP53, miR-34a, miR-34b, and miR-34c and increased protein levels of ZAP70. Low expression levels of ZAP70 have been found to be positively correlated with survival in patients with the indolent course of CLL carrying 13q deletions, and it was associated with increased TP53 levels and transactivation of miR-34b/ miR-34c. In this way, the authors found a microRNA/TP53 feedback circuitry associated with the pathogenesis and prognosis of CLL and revealed a new pathogenic model for human CLL.

As mentioned above, miR-29 expression correlated with the clinical course of CLL. Low expression of miR-29c was associated to patients who had a poor prognosis and shorter treatment-free survival as well as reduced overall survival (Calin \& Croce, 2009).

Thus, advances in the identification of miRNAs as CLL biomarkers, as well as the mode of regulation of gene expression and the pathway in which they are involved, should provide a useful prognostic tool for patient stratification and more appropriated treatments.

\subsection{Circulating miRNAs as novel biomarkers in CLL}

Nowadays, after the identification of extracellular circulating microRNAs in plasma microvesicles or ribonucleoprotein complexes (Cortez et al., 2011; Valadi et al., 2007), they have become an attractive source of new nucleic acid-based biomarkers. Today miRNAs are considered powerful markers for early detection, prognosis, response, and recurrence surveillance of different cancers because they are widely involved in oncogenesis (Taylor \& Gercel-Taylor, 2008). The diagnostic and prognostic potential of miRNAs as cancer biomarkers relies mainly on their high stability and resistance to storage handling. It has been consistently shown that serum miRNAs remain stable after being subjected to severe 
conditions that would normally degrade most RNAs. This stability can be partially explained by the discovery of lipoprotein complexes, including small membrane vesicles of endocytic origin called exosomes or microvesicles (30-1000 nm in diameter), containing miRNAs, mRNAs and proteins. Exosomes can be formed through inward budding of endosomal membranes, giving rise to intracellular multi vesicular bodies (MVBs) that later fuse with the plasma membrane, releasing the exosomes to the exterior (Figure 1). In contrast, microvesicles are originated by outward budding from the plasma membrane. The utility of miRNAs as diagnostic markers will be increased because samples of human plasma and serum can be obtained in a less invasive manner than can tissues.

Exosomes containing miRNAs were found in blood (Hunter et al., 2008), but also in other types of body fluids such as saliva and urine (Michael et al.). Importantly, exosomes represent a newly discovered mechanism by which donor cells can communicate and influence the gene expression of recipient cells. These findings were first demonstrated by the same study that discovered miRNAs in exosomes, in which mouse mast cell exosomes were added to human mast cells, leading to a subsequent detection of mouse proteins in the human cells (Valadi et al., 2007).

Tumor-specific miRNAs were first discovered in the serum of patients with diffuse large B cell lymphoma where high levels of miR-21 correlated with improved relapse-free survival (Lawrie et al., 2009). In an elegant experiment in a xenograft mouse prostate cancer model, the presence of circulating tumor-derived miR-629 and miR-660 was confirmed in blood with $100 \%$ sensitivity and specificity (Mitchell et al., 2008). In addition to showing that both serum and plasma samples are adequate for measuring specific miRNA levels, the investigators reported that by measuring serum levels of miR-141, they were able to distinguish patients with prostate cancer from healthy subjects. Since then, over 100 studies have assessed the potential use of serum or plasma miRNAs as biomarkers in different types of cancer (Cortez et al., 2011). In a comprehensive study, miRNA-expression profiles were identified in the sera of patients with lung or colorectal cancer, or diabetes by extracting miRNA from the serum (Chen et al., 2008). Although a unique expression profile of serum miRNAs was identified for each cancer type, an overlap was found in the profiles of specimens from all diseases analyzed in the study, including diabetes. In addition, this study also showed that miRNA-expression profiles differed between the serum and blood cells of lung cancer patients, while similar miRNA-expression profiles were seen in the serum and blood cells of healthy controls. These findings suggest that tumor-specific miRNAs in serum are derived not only from circulating blood cells but also cancer cells. An actualized compendium of relevant circulating miRNAs with potential as biomarkers for cancer was recently reviewed by Cortez et al. (Cortez et al., 2011).

Because most current approaches to cancer screening are invasive and unable to detect early-stage disease, it is important to determine when tumor-related circulating miRNAs can be detected in the bloodstream during disease development between other important factors. In this respect, it was recently reported that miRNAs can be also sensitive biomarkers for CLL, because certain extracellular miRNAs are present in CLL patient plasma at levels significantly different from healthy controls and from patients affected by other hematologic malignancies (Klein et al., 2010). Moreover, in this study the authors also determined that level of circulating miR-20a correlates reliably with diagnosis-to treatment time and miR-483-5p elevated almost six fold in plasma of CLL patients is predicted to target the mediators of IL-15 that induces CLL proliferation and drives CLL cell migration 
and infiltration. Although most of the miRNA-mRNA interactions are yet to be fully validated, the roles of these plasma miRNAs in CLL present intriguing biological questions with medically significant implications (Moussay et al., 2011; Ward et al., 2011).

\section{MiRNAs as predictors of CLL responses to therapy}

\subsection{Chemotherapy resistance}

In spite of the existence of effective treatments for patients suffering CLL, present therapeutic regimens are not totally effective and additional therapies are required.

Recently fludarabine-refractory CLL was linked to patients with p53 deletion and low miR34a expression. It was previously mentioned that miR-34a is positively regulated by p53. Low miR-34a expression levels were statistically significantly associated with impaired DNA damage response, p53 mutations, and fludarabine-refractory CLL either with or without p53 deletion (Calin \& Croce, 2009).

In addition, patients with resistant disease exhibited increased miR-181a and decreased miR-29a expression as consequence of their regulation by the myelocytomatosis viral oncogene homolog (MYC), which is increased in patients with fludarabine-resistant disease (Moussay et al., 2010).

These are the first identified miRNAs associated in the complex network of molecules associated to drug resistance and sensitivity in patients with CLL.

\subsection{Therapy}

According to the novel miRNAs described as biomarkers of prognosis and treatment in CLL, one of the most expecting use of them is the possible utility in the therapy of CLL.

The advantage to use these molecules for therapy, compared to other RNA inhibition strategies, relies on the fact that they are not only tiny and simple, but also they can target more than one mRNA relevant for the pathway of the disease (Calin \& Croce, 2009).

Different chemical modification as are 2'-O-methylphosphorothioate oligonucleotides, locked nucleic acid and miRNA sponge are currently being used to improve the blocking capacity and half-life of miRNA. In addition Liposome-oligonucleotide Complexes, among other strategies are been improved for the delivery, specificity and reduction of toxicity of miRNAs.

As for miR-122, which seems to be effective for the treatment hepatitis $C$ in ongoing clinical trials (Lanford et al.), the potential use of miRNAs in CLL treatment has only recently been envisioned and were only used in vitro.

\section{Concluding remarks}

In this chapter, we have analyzed the potential contribution of microRNAs as novel players and biomarkers in CLL pathogenesis.

The role of miRNAs as key regulatory molecules that control a wide variety of fundamental cellular processes, such as proliferation, death, differentiation, motility, invasiveness, etc., is 
increasingly recognized in almost all fields of biological and biomedical sciences. Over the last years, microRNAs have emerged as new actors in cancer biology as well as new diagnostic biomarkers and therapeutic targets in human cancer. This review attempts to briefly outline our current knowledge on the abnormalities of miRNAs found to be associated with CLL pathogenesis and possible mechanisms underlying the roles of miRNAs in CLL initiation and progression and to provide a perspective insight in using miRNAs as new CLL biomarkers. Nowadays, microRNAs are proposed as new sensitive, non-invasive and inexpensive biomarkers in CLL for early stage detection, predict outcome, monitor treatment and screen for disease recurrence. Understanding the significance of microRNAs in the pathogenesis of CLL represents an important dimension in miRNA research as it may lead to the development of miRNA-based novel therapeutic strategies or diagnostic/prognostic biomarkers. Additionally, microRNAs should also afford new avenues for exploring innovative pathways in CLL pathogenesis.

\section{References}

Aqeilan, R.I., Calin, G.A., and Croce, C.M. (2010). miR-15a and miR-16-1 in cancer: discovery, function and future perspectives. Cell death and differentiation 17, 215220.

Asslaber, D., Pinon, J.D., Seyfried, I., Desch, P., Stocher, M., Tinhofer, I., Egle, A., Merkel, O., and Greil, R. (2010). microRNA-34a expression correlates with MDM2 SNP309 polymorphism and treatment-free survival in chronic lymphocytic leukemia. Blood 115, 4191-4197.

Auer, R.L., Riaz, S., and Cotter, F.E. (2007). The 13q and 11q B-cell chronic lymphocytic leukaemia-associated regions derive from a common ancestral region in the zebrafish. British journal of haematology 137, 443-453.

Bonci, D., Coppola, V., Musumeci, M., Addario, A., Giuffrida, R., Memeo, L., D'Urso, L., Pagliuca, A., Biffoni, M., Labbaye, C., et al. (2008). The miR-15a-miR-16-1 cluster controls prostate cancer by targeting multiple oncogenic activities. Nature medicine $14,1271-1277$.

Calin, G.A., Cimmino, A., Fabbri, M., Ferracin, M., Wojcik, S.E., Shimizu, M., Taccioli, C., Zanesi, N., Garzon, R., Aqeilan, R.I., et al. (2008). MiR-15a and miR-16-1 cluster functions in human leukemia. Proceedings of the National Academy of Sciences of the United States of America 105, 5166-5171.

Calin, G.A., and Croce, C.M. (2009). Chronic lymphocytic leukemia: interplay between noncoding RNAs and protein-coding genes. Blood 114, 4761-4770.

Calin, G.A., Dumitru, C.D., Shimizu, M., Bichi, R., Zupo, S., Noch, E., Aldler, H., Rattan, S., Keating, M., Rai, K., et al. (2002). Frequent deletions and down-regulation of microRNA genes miR15 and miR16 at 13q14 in chronic lymphocytic leukemia. Proceedings of the National Academy of Sciences of the United States of America 99, 15524-15529.

Calin, G.A., Ferracin, M., Cimmino, A., Di Leva, G., Shimizu, M., Wojcik, S.E., Iorio, M.V., Visone, R., Sever, N.I., Fabbri, M., et al. (2005). A MicroRNA signature associated with prognosis and progression in chronic lymphocytic leukemia. The New England journal of medicine 353, 1793-1801.

Calin, G.A., Liu, C.G., Sevignani, C., Ferracin, M., Felli, N., Dumitru, C.D., Shimizu, M., Cimmino, A., Zupo, S., Dono, M., et al. (2004a). MicroRNA profiling reveals distinct signatures in B cell chronic lymphocytic leukemias. Proceedings of the National Academy of Sciences of the United States of America 101, 11755-11760. 
Calin, G.A., Sevignani, C., Dumitru, C.D., Hyslop, T., Noch, E., Yendamuri, S., Shimizu, M., Rattan, S., Bullrich, F., Negrini, M., et al. (2004b). Human microRNA genes are frequently located at fragile sites and genomic regions involved in cancers. Proceedings of the National Academy of Sciences of the United States of America 101, 2999-3004.

Cimmino, A., Calin, G.A., Fabbri, M., Iorio, M.V., Ferracin, M., Shimizu, M., Wojcik, S.E., Aqeilan, R.I., Zupo, S., Dono, M., et al. (2005). miR-15 and miR-16 induce apoptosis by targeting BCL2. Proceedings of the National Academy of Sciences of the United States of America 102, 13944-13949.

Cole, K.A., Attiyeh, E.F., Mosse, Y.P., Laquaglia, M.J., Diskin, S.J., Brodeur, G.M., and Maris, J.M. (2008). A functional screen identifies miR-34a as a candidate neuroblastoma tumor suppressor gene. Mol Cancer Res 6, 735-742.

Corney, D.C., Flesken-Nikitin, A., Godwin, A.K., Wang, W., and Nikitin, A.Y. (2007). MicroRNA-34b and MicroRNA-34c are targets of p53 and cooperate in control of cell proliferation and adhesion-independent growth. Cancer research 67, 8433-8438.

Cortez, M.A., Bueso-Ramos, C., Ferdin, J., Lopez-Berestein, G., Sood, A.K., and Calin, G.A. (2011). MicroRNAs in body fluids--the mix of hormones and biomarkers. Nat Rev Clin Oncol 8, 467-477.

Croce, C.M. (2009). Causes and consequences of microRNA dysregulation in cancer. Nature reviews 10, 704-714.

Chen, X., Ba, Y., Ma, L., Cai, X., Yin, Y., Wang, K., Guo, J., Zhang, Y., Chen, J., Guo, X., et al. (2008). Characterization of microRNAs in serum: a novel class of biomarkers for diagnosis of cancer and other diseases. Cell Res 18, 997-1006.

Chu, C.Y., and Rana, T.M. (2007). Small RNAs: regulators and guardians of the genome. Journal of cellular physiology 213, 412-419.

Deng, S., Calin, G.A., Croce, C.M., Coukos, G., and Zhang, L. (2008). Mechanisms of microRNA deregulation in human cancer. Cell cycle (Georgetown, Tex 7, 2643-2646.

Fabbri, M., Bottoni, A., Shimizu, M., Spizzo, R., Nicoloso, M.S., Rossi, S., Barbarotto, E., Cimmino, A., Adair, B., Wojcik, S.E., et al. (2011). Association of a microRNA/TP53 feedback circuitry with pathogenesis and outcome of B-cell chronic lymphocytic leukemia. Jama 305, 59-67.

Fabbri, M., Garzon, R., Cimmino, A., Liu, Z., Zanesi, N., Callegari, E., Liu, S., Alder, H., Costinean, S., Fernandez-Cymering, C., et al. (2007). MicroRNA-29 family reverts aberrant methylation in lung cancer by targeting DNA methyltransferases $3 \mathrm{~A}$ and 3B. Proceedings of the National Academy of Sciences of the United States of America 104, 15805-15810.

Farazi, T.A., Spitzer, J.I., Morozov, P., and Tuschl, T. (2011). miRNAs in human cancer. The Journal of pathology 223, 102-115.

Frenquelli, M., Muzio, M., Scielzo, C., Fazi, C., Scarfo, L., Rossi, C., Ferrari, G., Ghia, P., and Caligaris-Cappio, F. (2010). MicroRNA and proliferation control in chronic lymphocytic leukemia: functional relationship between miR-221/222 cluster and p27. Blood 115, 3949-3959.

Fulci, V., Chiaretti, S., Goldoni, M., Azzalin, G., Carucci, N., Tavolaro, S., Castellano, L., Magrelli, A., Citarella, F., Messina, M., et al. (2007). Quantitative technologies establish a novel microRNA profile of chronic lymphocytic leukemia. Blood 109, 4944-4951.

Garzon, R., Calin, G.A., and Croce, C.M. (2009). MicroRNAs in Cancer. Annual review of medicine 60, 167-179.

Hanlon, K., Rudin, C.E., and Harries, L.W. (2009). Investigating the targets of MIR-15a and MIR-16-1 in patients with chronic lymphocytic leukemia (CLL). PloS one 4, e7169. 
He, L., Thomson, J.M., Hemann, M.T., Hernando-Monge, E., Mu, D., Goodson, S., Powers, S., Cordon-Cardo, C., Lowe, S.W., Hannon, G.J., et al. (2005). A microRNA polycistron as a potential human oncogene. Nature 435, 828-833.

Hermeking, H. (2010). The miR-34 family in cancer and apoptosis. Cell death and differentiation 17, 193-199.

Hunter, M.P., Ismail, N., Zhang, X., Aguda, B.D., Lee, E.J., Yu, L., Xiao, T., Schafer, J., Lee, M.L., Schmittgen, T.D., et al. (2008). Detection of microRNA expression in human peripheral blood microvesicles. PloS one 3, e3694.

Klein, U., and Dalla-Favera, R. (2010). New insights into the pathogenesis of chronic lymphocytic leukemia. Seminars in cancer biology 20, 377-383.

Klein, U., Lia, M., Crespo, M., Siegel, R., Shen, Q., Mo, T., Ambesi-Impiombato, A., Califano, A., Migliazza, A., Bhagat, G., et al. (2010). The DLEU2/miR-15a/16-1 cluster controls B cell proliferation and its deletion leads to chronic lymphocytic leukemia. Cancer cell 17, 28-40.

Lagos-Quintana, M., Rauhut, R., Lendeckel, W., and Tuschl, T. (2001). Identification of novel genes coding for small expressed RNAs. Science (New York, NY 294, 853-858.

Lanford, R.E., Hildebrandt-Eriksen, E.S., Petri, A., Persson, R., Lindow, M., Munk, M.E., Kauppinen, S., and Orum, H. Therapeutic silencing of microRNA-122 in primates with chronic hepatitis C virus infection. Science (New York, NY 327, 198-201.

Lawrie, C.H., Ballabio, E., Dyar, O.J., Jones, M., Ventura, R., Chi, J., Tramonti, D., Gooding, S., Boultwood, J., Wainscoat, J.S., et al. (2009). MicroRNA expression in chronic lymphocytic leukaemia. British journal of haematology 147, 398-402.

Linsley, P.S., Schelter, J., Burchard, J., Kibukawa, M., Martin, M.M., Bartz, S.R., Johnson, J.M., Cummins, J.M., Raymond, C.K., Dai, H., et al. (2007). Transcripts targeted by the microRNA-16 family cooperatively regulate cell cycle progression. Molecular and cellular biology 27, 2240-2252.

Marton, S., Garcia, M.R., Robello, C., Persson, H., Trajtenberg, F., Pritsch, O., Rovira, C., Naya, H., Dighiero, G., and Cayota, A. (2008). Small RNAs analysis in CLL reveals a deregulation of miRNA expression and novel miRNA candidates of putative relevance in CLL pathogenesis. Leukemia 22, 330-338.

Michael, A., Bajracharya, S.D., Yuen, P.S., Zhou, H., Star, R.A., Illei, G.G., and Alevizos, I. Exosomes from human saliva as a source of microRNA biomarkers. Oral diseases 16, 34-38.

Migliazza, A., Cayanis, E., Bosch-Albareda, F., Komatsu, H., Martinotti, S., Toniato, E., Kalachikov, S., Bonaldo, M.F., Jelene, P., Ye, X., et al. (2000). Molecular pathogenesis of B-cell chronic lymphocytic leukemia: analysis of 13q14 chromosomal deletions. Current topics in microbiology and immunology 252, 275-284.

Mitchell, P.S., Parkin, R.K., Kroh, E.M., Fritz, B.R., Wyman, S.K., Pogosova-Agadjanyan, E.L., Peterson, A., Noteboom, J., O'Briant, K.C., Allen, A., et al. (2008). Circulating microRNAs as stable blood-based markers for cancer detection. Proceedings of the National Academy of Sciences of the United States of America 105, 10513-10518.

Mott, J.L., Kobayashi, S., Bronk, S.F., and Gores, G.J. (2007). mir-29 regulates Mcl-1 protein expression and apoptosis. Oncogene 26, 6133-6140.

Moussay, E., Palissot, V., Vallar, L., Poirel, H.A., Wenner, T., El Khoury, V., Aouali, N., Van Moer, K., Leners, B., Bernardin, F., et al. (2010). Determination of genes and microRNAs involved in the resistance to fludarabine in vivo in chronic lymphocytic leukemia. Molecular cancer 9, 115.

Moussay, E., Wang, K., Cho, J.H., van Moer, K., Pierson, S., Paggetti, J., Nazarov, P.V., Palissot, V., Hood, L.E., Berchem, G., et al. (2011). MicroRNA as biomarkers and 
regulators in B-cell chronic lymphocytic leukemia. Proceedings of the National Academy of Sciences of the United States of America 108, 6573-6578.

Nana-Sinkam, S.P., and Croce, C.M. (2010). MicroRNA in chronic lymphocytic leukemia: transitioning from laboratory-based investigation to clinical application. Cancer genetics and cytogenetics 203, 127-133.

Palamarchuk, A., Efanov, A., Nazaryan, N., Santanam, U., Alder, H., Rassenti, L., Kipps, T., Croce, C.M., and Pekarsky, Y. (2010). 13q14 deletions in CLL involve cooperating tumor suppressors. Blood 115, 3916-3922.

Pekarsky, Y., Santanam, U., Cimmino, A., Palamarchuk, A., Efanov, A., Maximov, V., Volinia, S., Alder, H., Liu, C.G., Rassenti, L., et al. (2006). Tcl1 expression in chronic lymphocytic leukemia is regulated by miR-29 and miR-181. Cancer research 66, 11590-11593.

Rossi, S., Shimizu, M., Barbarotto, E., Nicoloso, M.S., Dimitri, F., Sampath, D., Fabbri, M., Lerner, S., Barron, L.L., Rassenti, L.Z., et al. (2010). microRNA fingerprinting of CLL patients with chromosome $17 \mathrm{p}$ deletion identify a miR-21 score that stratifies early survival. Blood 116, 945-952.

Rovira, C., Guida, M.C., and Cayota, A. (2010). MicroRNAs and other small silencing RNAs in cancer. IUBMB life 62, 859-868.

Sanchez-Beato, M., Sanchez-Aguilera, A., and Piris, M.A. (2003). Cell cycle deregulation in B-cell lymphomas. Blood 101, 1220-1235.

Santanam, U., Zanesi, N., Efanov, A., Costinean, S., Palamarchuk, A., Hagan, J.P., Volinia, S., Alder, H., Rassenti, L., Kipps, T., et al. (2010). Chronic lymphocytic leukemia modeled in mouse by targeted miR-29 expression. Proceedings of the National Academy of Sciences of the United States of America 107, 12210-12215.

Stamatopoulos, B., Meuleman, N., Haibe-Kains, B., Saussoy, P., Van Den Neste, E., Michaux, L., Heimann, P., Martiat, P., Bron, D., and Lagneaux, L. (2009). microRNA-29c and microRNA-223 down-regulation has in vivo significance in chronic lymphocytic leukemia and improves disease risk stratification. Blood 113, 5237-5245.

Taylor, D.D., and Gercel-Taylor, C. (2008). MicroRNA signatures of tumor-derived exosomes as diagnostic biomarkers of ovarian cancer. Gynecologic oncology 110, 13-21.

Valadi, H., Ekstrom, K., Bossios, A., Sjostrand, M., Lee, J.J., and Lotvall, J.O. (2007). Exosome-mediated transfer of mRNAs and microRNAs is a novel mechanism of genetic exchange between cells. Nature cell biology 9, 654-659.

Vasilatou, D., Papageorgiou, S., Pappa, V., Papageorgiou, E., and Dervenoulas, J. (2010). The role of microRNAs in normal and malignant hematopoiesis. European journal of haematology 84, 1-16.

Visone, R., Rassenti, L.Z., Veronese, A., Taccioli, C., Costinean, S., Aguda, B.D., Volinia, S., Ferracin, M., Palatini, J., Balatti, V., et al. (2009). Karyotype-specific microRNA signature in chronic lymphocytic leukemia. Blood 114, 3872-3879.

Ward, B.P., Tsongalis, G.J., and Kaur, P. (2011). MicroRNAs in chronic lymphocytic leukemia. Experimental and molecular pathology 90, 173-178.

Wright, F.A., Lemon, W.J., Zhao, W.D., Sears, R., Zhuo, D., Wang, J.P., Yang, H.Y., Baer, T., Stredney, D., Spitzner, J., et al. (2001). A draft annotation and overview of the human genome. Genome biology 2, RESEARCH0025.

Zhao, J.J., Lin, J., Lwin, T., Yang, H., Guo, J., Kong, W., Dessureault, S., Moscinski, L.C., Rezania, D., Dalton, W.S., et al. (2010) microRNA expression profile and identification of miR-29 as a prognostic marker and pathogenetic factor by targeting CDK6 in mantle cell lymphoma. Blood 115, 2630-2639. 


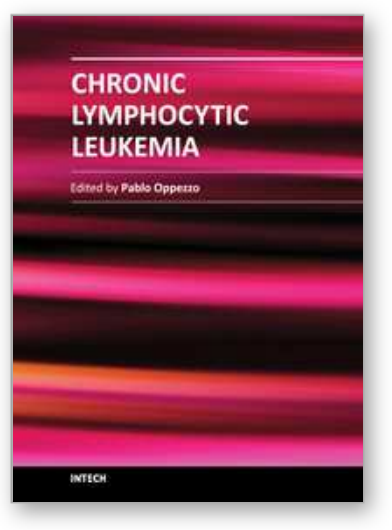

\author{
Chronic Lymphocytic Leukemia \\ Edited by Dr. Pablo Oppezzo
}

ISBN 978-953-307-881-6

Hard cover, 448 pages

Publisher InTech

Published online 10, February, 2012

Published in print edition February, 2012

B-cell chronic lymphocytic leukemia (CLL) is considered a single disease with extremely variable course, and survival rates ranging from months to decades. It is clear that clinical heterogeneity reflects biologic diversity with at least two major subtypes in terms of cellular proliferation, clinical aggressiveness and prognosis. As CLL progresses, abnormal hematopoiesis results in pancitopenia and decreased immunoglobulin production, followed by nonspecific symptoms such as fatigue or malaise. A cure is usually not possible, and delayed treatment (until symptoms develop) is aimed at lengthening life and decreasing symptoms. Researchers are playing a lead role in investigating CLL's cause and the role of genetics in the pathogenesis of this disorder. Research programs are dedicated towards understanding the basic mechanisms underlying CLL with the hope of improving treatment options.

\title{
How to reference
}

In order to correctly reference this scholarly work, feel free to copy and paste the following:

Maria Rosa Garcia-Silva, Maria Catalina Güida and Alfonso Cayota (2012). Contribution of microRNAs to CLL Biology and Their Potential as New Biomarkers, Chronic Lymphocytic Leukemia, Dr. Pablo Oppezzo (Ed.), ISBN: 978-953-307-881-6, InTech, Available from: http://www.intechopen.com/books/chronic-lymphocyticleukemia/contribution-of-micrornas-to-cll-biology-and-their-potential-as-new-biomarkers

\section{INTECH}

open science | open minds

\author{
InTech Europe \\ University Campus STeP Ri \\ Slavka Krautzeka 83/A \\ 51000 Rijeka, Croatia \\ Phone: +385 (51) 770447 \\ Fax: +385 (51) 686166 \\ www.intechopen.com
}

\author{
InTech China \\ Unit 405, Office Block, Hotel Equatorial Shanghai \\ No.65, Yan An Road (West), Shanghai, 200040, China \\ 中国上海市延安西路65号上海国际贵都大饭店办公楼 405 单元 \\ Phone: +86-21-62489820 \\ Fax: $+86-21-62489821$
}


(C) 2012 The Author(s). Licensee IntechOpen. This is an open access article distributed under the terms of the Creative Commons Attribution 3.0 License, which permits unrestricted use, distribution, and reproduction in any medium, provided the original work is properly cited. 